\title{
Retarder le mariage précoce chez les filles défavorisées en milieu rural dans la région Amhara, en Éthiopie, grâce au soutien social, à l'éducation et à la sensibilisation des communautés
}

Eunice N. Muthengi

Population Council

Annabel Erulkar

Population Council

Follow this and additional works at: https://knowledgecommons.popcouncil.org/departments_sbsr-pgy

Part of the Demography, Population, and Ecology Commons, Family, Life Course, and Society

Commons, Gender and Sexuality Commons, and the International Public Health Commons

How does access to this work benefit you? Let us know!

\section{Recommended Citation}

Muthengi, Eunice N. and Annabel Erulkar. 2011. "Retarder le mariage précoce chez les filles défavorisées en milieu rural dans la région Amhara, en Éthiopie, grâce au soutien social, à l'éducation et à la sensibilisation des communautés," Promouvoir la santé, la sécurité et la productivité transitions vers l'âge adulte Bulletin no. 20. New York: Population Council. 


\section{Retarder le mariage précoce chez les filles défavorisées en milieu rural dans la région Amhara, en Éthiopie, grâce au soutien social, à l'éducation et à la sensibilisation des communautés}

\section{Preparé par Eunice Muthengi et Annabel Erulkar}

'Éthiopie affiche des taux extrêmement élevés de mariages d'enfants, en particulier dans les zones rurales, et subit une épidémie de VIH qui touche les femmes de façon disproportionnée. En Éthiopie, 44 pour cent de la population est âgée de moins de 15 ans et les programmes de santé publique à l'attention des adolescents se sont multipliés. Ces programmes sont essentiellement situés dans les zones urbaines et ciblent les adolescents scolarisés, les garçons adolescents plus âgés et les jeunes hommes, ainsi que les adolescents célibataires qui vivent avec leurs parents. La plupart des programmes négligent la grande majorité des jeunes (85 pour cent) qui vivent dans des zones rurales et les principales catégories de filles vulnérables-les filles non scolarisées, les filles à risque de mariage précoce et les filles qui sont déjà mariées (Erulkar et al 2004 ; MOFED 2005).

Dans la région rurale de l'Amhara, 50 pour cent des filles sont mariées avant l'âge de 15 ans et plus de 80 pour cent sont mariées avant l'âge de 18 ans (Erulkar et al 2010). Le mariage des enfants ou le mariage précoce, défini comme un mariage survenant avant l'âge de 18 ans, est considéré comme une violation selon de nombreuses normes nationales et internationales, et est illégal en Éthiopie. L'article 7 du Code révisé de la famille en Éthiopie stipule que l'âge légal du mariage est de 18 ans pour les hommes et les femmes. Le mariage à un jeune âge limite les possibilités de scolarisation, d'acquisition des compétences et de développement personnel des filles (Mensch et al 1998). Il augmente également le risque d'une grossesse précoce, qui est associée à des conséquences néfastes sur la santé comme la mortalité maternelle, l'anémie, les complications obstétricales, et d'autres issues défavorables (Makinson 1985 ; Adhikari 2003 ; Haberland et al 2005 ; Nour 2006).

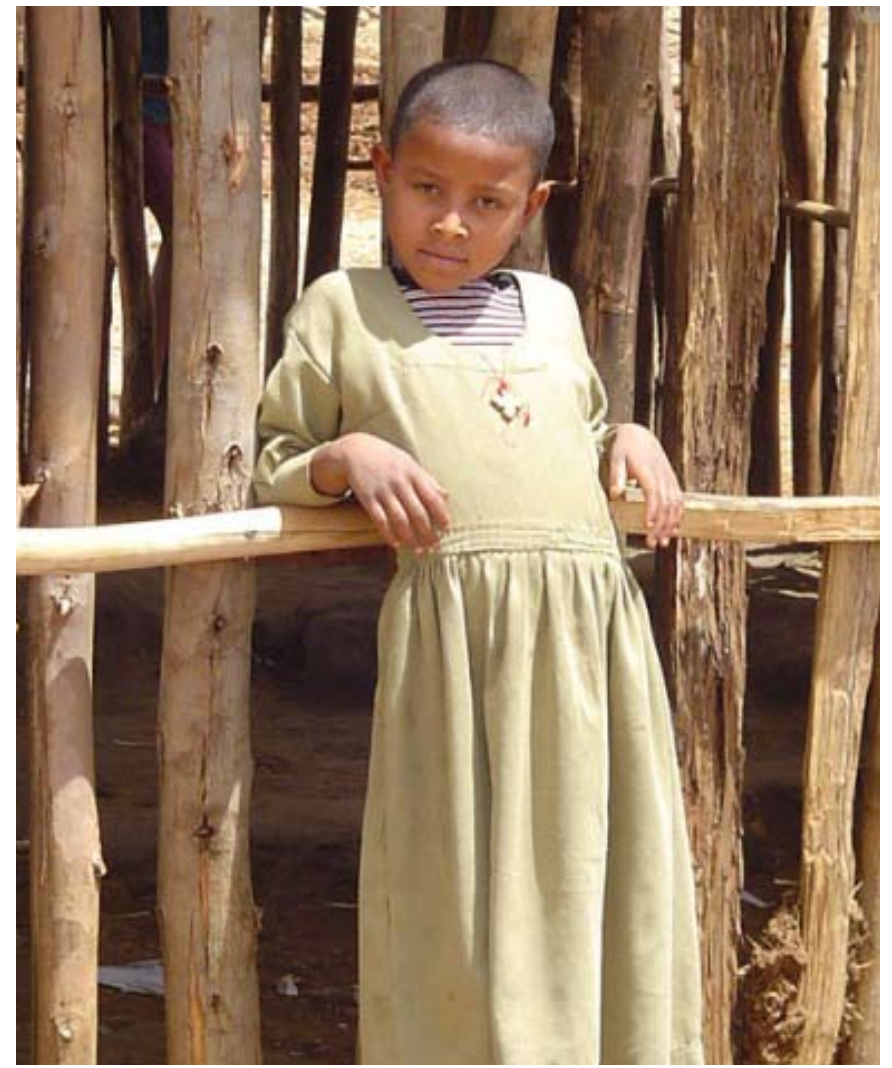

Afin de mieux comprendre la vie des adolescentes mariées en Éthiopie rurale, y compris leurs atouts sociaux, la scolarisation et le rôle du mariage, des chercheurs ont réalisé une enquête auprès de plus de 1800 adolescentes de la région Amhara (Erulkar et al 2004). Sur la base des conclusions de cette étude, en 2004, le Bureau régio- 
nal de la Jeunesse et des Sports d'Amhara et le Population Council ont créé le projet Berhane Hewan pour aider les filles mariées et célibataires. L'objectif du projet Berhane Hewan, qui signifie « Lumière pour Ève » en amharique, est la mise en place de systèmes appropriés et efficaces pour protéger les jeunes filles à risque de mariage précoce et soutenir les adolescentes qui sont déjà mariées. Le programme Berhane Hewan comprend quatre composantes : (1) une formation de groupe dispensée par des tutrices adultes, y compris des groupes pour les filles mariées ; (2) un appui en faveur de la scolarisation des filles grâce à la fourniture de matériel scolaire de base, ainsi qu'un soutien pour la participation volontaire dans l'éducation informelle (alphabétisation de base et calcul) ; (3) des « conversations communautaires » visant à engager la communauté dans les questions clés, telles que le mariage précoce, et la résolution collective des problèmes ; et (4) des mesures incitatives ou le versement de primes financières soumises à condition aux familles qui maintiennent les filles à l'école et qui n'ont pas recours au mariage précoce.

Entre 2004 et 2006, une intervention quasi-expérimentale a été menée par le Population Council dans la région rurale de l'Amhara dans le but de comparer les résultats entre les filles vivant dans la zone du programme du village de Mosebo et celles vivant dans la zone témoin du village d'Enamirt. Une évaluation de l'intervention apporte la preuve que le mariage précoce peut être évité grâce à un programme qui aborde simultanément les facteurs économiques et sociaux qui favorisent le mariage précoce et améliorent l'accès à la scolarisation des filles (Erulkar et Muthengi 2009).

\section{Améliorations dans l'éducation formelle et non formelle} Les résultats de l'évaluation du projet Berhane Hewan indiquent que les chances de bénéficier d'une éducation formelle et informelle aug-

Figure 1 Fréquentation scolaire avant et après le projet pilote

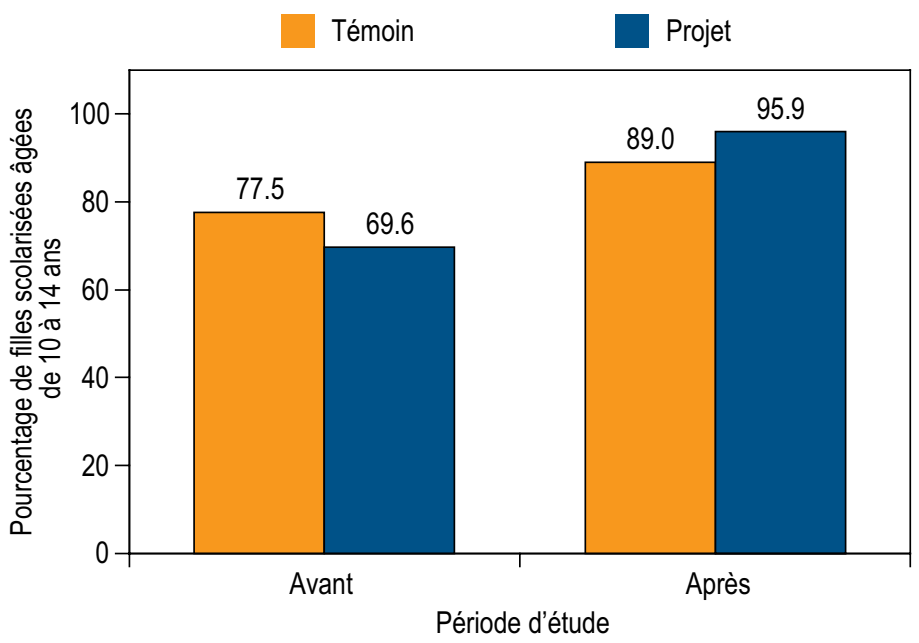

mentent tant pour les filles mariées que pour les filles célibataires. Les filles qui étaient encore à l'école ou celles qui voulaient rejoindre des classes formelles ont été aidées dans la poursuite de leur éducation. Du matériel scolaire de base, tels que cahiers, stylos et crayons, a été fourni aux filles. Cette aide, quoique modeste -environ 4 dollars en fournitures par fille et par année- représentait le niveau soutien nécessaire pour que de nombreuses familles rurales pauvres maintiennent leurs filles à l'école. L'éducation non formelle, (y compris l'alphabétisation de base et les leçons de calcul), a également été proposée aux jeunes filles qui ne souhaitaient pas retourner à l'école ou qui n'avaient jamais reçu d'éducation formelle.

Une amélioration significative de la fréquentation scolaire et des niveaux d'alphabétisation a été observée dans la zone du projet chez les filles âgées entre 10 et 14 ans. Les filles qui vivaient dans la zone d'étude au village de Mosebo étaient plus susceptibles de commencer une éducation formelle et d'être scolarisées à la fin de l'intervention. La proportion de filles scolarisées était légèrement plus élevée dans la zone témoin du village d'Enamirt avant l'intervention (70 pour cent dans la zone du projet contre 78 pour cent dans la zone témoin), mais à la fin de l'intervention, les filles de Mosebo étaient significativement plus susceptibles d'être scolarisées (96 pour cent dans la zone du projet contre 89 pour cent dans la zone témoin) (voir la figure 1). Bien que les filles vivant dans la zone du projet étaient plus susceptibles d'être analphabètes avant l'intervention (45 pour cent contre 28 pour cent dans la zone témoin), les niveaux étaient identiques à la fin du programme, tant dans la zone d'étude que dans la zone témoin (21 pour cent contre 19 pour cent respectivement).

Amélioration des connaissances en matière de soutien social et de santé de la reproduction

Les filles âgées entre 10 et 19 ans et éligibles au programme ont été recrutées par les tuteurs du programme et organisées en groupes d'environ 15 à 20 jeunes filles mariées ou célibataires. Les filles mariées se réunissaient une fois par semaine en raison de leurs contraintes de temps, alors que les filles célibataires se réunissaient cinq jours par semaine. Lors des réunions de groupe, les thèmes proposés aux filles couvraient la santé reproductive, l'accès aux services de planification familiale et aux compétences de subsistance telles que les techniques agricoles, l'élevage de volaille, et la construction d'articles ménagers. En outre, les filles qui souhaitaient recourir à la planification familiale bénéficiaient d'une carte médicale leur permettant de consulter dans un établissement public local (les services et les fournitures relatifs à la planification familiale sont gratuits dans les établissements publics, et le coût de la carte médicale, soit 20 birr ou l'équivalent de 2,20 dollars, était financé par le projet.

Les résultats de l'évaluation indiquent que le programme a joué un rôle clé dans l'expansion des réseaux sociaux pour les filles vivant 
Figure 2 Âge du premier mariage par zone de résidence, mesuré à la fin de l'étude

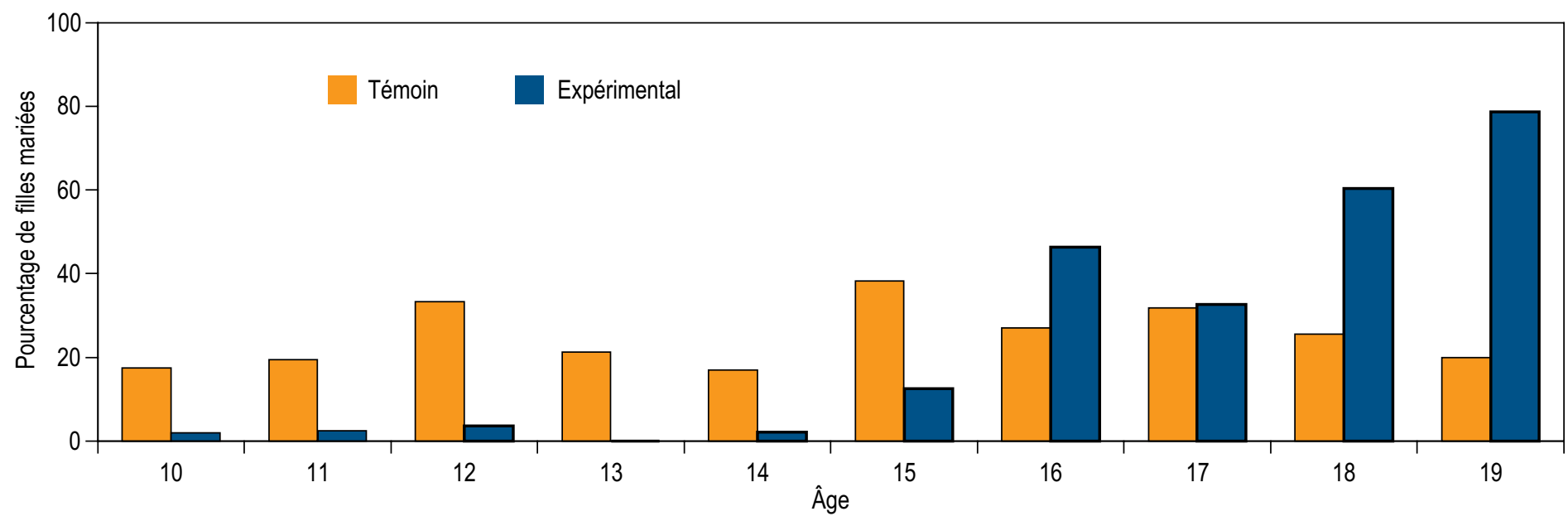

dans la zone d'étude de Mosebo. Au début du programme, seulement 30 pour cent des filles vivant dans la zone d'étude avaient indiqué qu'elles avaient une meilleure amie qui n'était pas membre de leur famille comparé à 48 pour cent des filles dans la zone témoin. À la fin du programme, cependant, environ la moitié des filles dans les deux zones avaient une meilleure amie qui n'était pas membre de leur famille. La proportion des filles qui ont déclaré parler à leurs amies les plus proches sur les différents thèmes de santé reproductive était inférieure ou équivalente dans la zone d'étude que dans la zone témoin avant l'intervention. À la fin du programme, les filles vivant dans la zone d'étude étaient plus susceptibles de parler à leurs amies de la planification familiale, de leurs problèmes de couples et des infections sexuellement transmissibles que les autres. Les leçons sur la santé reproductive au cours des réunions de groupe ont contribué à une augmentation significative des connaissances sur la santé reproductive et l'utilisation de contraceptifs. À la fin du programme, près des trois quarts des adolescentes sexuellement actives dans la zone du projet avaient utilisé une méthode contraceptive, contre moins de la moitié des filles dans la zone témoin.

\section{Retarder l'âge du mariage}

Deux composantes supplémentaires du programme ciblaient directement le fait de retarder l'âge du mariage : les conversations communautaires et les incitations financières. Des réunions de quartier ont eu lieu toutes les deux semaines à cinq endroits de la zone du programme, atteignant environ 70 membres de la communauté. Quatre animateurs formés ont mené des discussions sur le mariage précoce et les autres pratiques traditionnelles nuisibles, ainsi que sur des questions concernant les jeunes femmes et les filles. Cette technique de dialogue participatif a permis aux participants d'explorer les problèmes ensemble et de trouver des solutions communes. Après plusieurs mois de discussion, les participants ont décidé de mener des actions collectives (par exemple, éviter le mariage précoce), ont formé des sous-comités et ont tenté de convaincre les membres de leur communauté à adopter les mêmes décisions.

Des incitations financières ont été proposées aux parents et aux tuteurs des filles qui avaient participé au programme. Une chèvre d'une valeur d'environ 180 birr (20 dollars) leur a été proposée à la fin du programme (conjointement à une fille et sa famille) à condition de laisser les filles participer à au moins 80 pour cent des séances de groupe ou des séances scolaires et de s'engager à ne pas marier les filles pendant les deux années que durait le programme.

Les résultats indiquent que le programme a réussi à retarder l'âge du mariage chez les jeunes filles, mais pas chez les filles plus âgées. Avant la période d'étude, aucune différence significative n'avait été observée entre la proportion de filles qui avaient déjà été mariées dans la zone du projet et dans la zone témoin (10 pour cent contre 14 pour cent, respectivement). À la fin du programme, les filles âgées entre 10 et 14 ans étaient moins susceptibles de se marier dans la zone d'étude (2 pour cent) par rapport aux filles vivant dans la zone témoin (22 pour cent). Parmi les filles âgées de 15 à 19 ans, la proportion des filles mariées était identique dans les deux zones avant le programme, mais plus élevée dans la zone d'étude après l'intervention. Compte tenu de la courte période d'intervention, la tendance présentée dans la figure 2 semble refléter un retard de l'âge du mariage vers la fin de l'adolescence ; moins de filles ont été mariées avant l'âge de 15 ans, et plus de filles ont été mariées entre 16 et 19 ans.

\section{Le succès du projet Berhane Hewan}

Le fait de retarder l'âge du mariage pendant l'adolescence permet aux filles de profiter des années essentielles pendant lesquelles elles peuvent acquérir des compétences et se développer en tant qu'individus. 
La participation au projet pilote Berhane Hewan en Éthiopie rurale a amélioré la vie des jeunes filles en les aidant à rester à l'école ou apprendre à lire, en renforçant leurs réseaux sociaux, en améliorant leurs connaissances sur la santé reproductive, l'utilisation de la contraception, et en retardant l'âge de leur mariage. La réussite de ce programme représente un modèle pour des programmes identiques en Éthiopie et dans d'autres zones rurales où de nombreuses filles sont mariées avant l'âge de 15 ans et ne sont pas scolarisées. Les décideurs politiques et les responsables de programmes qui mettent en œuvre de tels programmes doivent :

- se pencher sur les motivations économiques poussant au mariage précoce en fournissant des incitations pour retarder l'âge du mariage ;

- investir dans l'éducation en augmentant les chances pour les filles de suivre une scolarité formelle et informelle ;

- élaborer des programmes de soutien pour lutter contre l'isolement des adolescentes mariées et célibataires ;

- fournir aux adolescentes l'accès aux services de planification familiale et aux informations relatives à la santé reproductive ; et

- sensibiliser les membres de la communauté sur les dangers du mariage précoce et des autres pratiques traditionnelles néfastes qui touchent les adolescentes.

Des programmes tels que Berhane Hewan peuvent offrir aux filles des alternatives au mariage grâce à l'éducation en traitant simultanément les facteurs économiques et sociaux qui favorisent le mariage précoce.

\section{Bibliographie}

Erulkar, Annabel S., Ferede Abebaw, Worku Ambelu, Woldemariam Girma, et al. 2010. "Ethiopia gender survey: A study in seven regions." New York: Population Council.

Erulkar, A.S., T. Mekbib, N. Simie, et T. Gulema. 2004. "The experience of adolescence in rural Amhara region, Ethiopia." Accra: Population Council.

Erulkar, A.S. et E. Muthengi. 2009. "Evaluation of Berhane Hewan: A program to delay child marriage in rural Ethiopia". International Perspectives on Sexual and Reproductive Health 35(1): 6-14

Haberland, N., E.L Chong, H.J. Bracken, et C. Parker. 2005. Early marriage and adolescent girls, YouthLens on Reproductive Health and HIV/AIDS. Research Triangle Park, NC: YouthNet, No. 15.

Makinson, C. 1985. "The health consequences of teenage fertility". Family Planning Perspectives. 17(3):132-139.

Mensch, B.S., J. Bruce, et M. Greene. 1998. The Uncharted Passage: Girls' Adolescence in the Developing World. New York: Population Council.

Ministère des Finances et du Développement économique (MOFED). 2005. Building on Progress: A Plan for Accelerated and Sustained Development to End Poverty (PASDEP), 2005/06-2009/10.

Nour, N.M. 2006. "Health consequences of child marriage in Africa". Emerging Infectious Diseases 12(11):1644-1649.

\section{Bailleurs de fonds}

Le Département du développement international (DFID) ; UNFPA, Fondation Nike, Fondation des Nations unies

Organisations partenaires

Ministère éthiopien des Femmes, de l'Enfance et de la Jeunesse ; Bureaux régionaux des Enfants et de la Jeunesse de l'Amhara

Pour plus d'informations, veuillez contacter :

\begin{tabular}{|c|c|c|c|}
\hline $\begin{array}{l}\text { W/ro Zimam Assefa } \\
\text { Head of Bureau } \\
\text { Regional Bureau of Women, } \\
\text { Children \& Youth } \\
\text { P.O. Box } 25 \\
\text { Bahir Dar ÉTHIOPIE }\end{array}$ & $\begin{array}{l}\text { Ato Tesfahun Wondie } \\
\text { Regional Bureau of Women, } \\
\text { Children \& Youth } \\
\text { P.O. Box } 25 \\
\text { Bahir Dar ÉTHIOPIE }\end{array}$ & $\begin{array}{l}\text { Sisay Melesse } \\
\text { Population Council } \\
\text { P.O. Box 25562-code } 1000 \\
\text { Addis Abeba ÉTHIOPIE }\end{array}$ & $\begin{array}{l}\text { Annabel Erulkar } \\
\text { Country Director/Senior Associate } \\
\text { Population Council } \\
\text { P.O. Box 25562-code } 1000 \\
\text { Addis Abeba ÉTHIOPIE } \\
\text { aerulkar@popcouncil.org }\end{array}$ \\
\hline
\end{tabular}

(2) Population Council

Le Population Council fait évoluer les perspectives sur les questions essentielles de santé et de développement. Nous cherchons à comprendre les causes et les conséquences de l'inégalité entre les sexes et des disparités sociales qui s'installent au cours de l'adolescence. Nous fournissons des données en vue d'améliorer les programmes et les politiques mis en place sur le terrain qui permettent d'assurer des transitions efficaces et productives vers la vie adulte dans les pays en développement. www.popcouncil.org

C) 2011 The Population Council, Inc.

4 - Rendez-vous sur www.popcouncil.org/publications/serialsbriefs/TABriefs.asp pour consulter tous les bulletins d'information de la série Promoting healthy, safe, and productive transitions to adulthood [Promouvoir des transitions saines, sécurisées et productives vers l'âge adulte]. 